\title{
VARIABILIDADE ESPACIAL DE ATRIBUTOS MINERALÓGICOS DE UM LATOSSOLO SOB DIFERENTES FORMAS DO RELEVO. II - CORRELAÇÃO ESPACIAL ENTRE MINERALOGIA E AGREGADOS ${ }^{(1)}$
}

\author{
Livia Arantes Camargo(2), José Marques Júnior ${ }^{(3)}$, Gener Tadeu \\ Pereira $^{(4)}$ \& Renata Alves Horvat ${ }^{(5)}$
}

\begin{abstract}
RESUMO
A caulinita (Ct), a gibbsita (Gb), a goethita (Gt) e a hematita (Hm) são os principais minerais da fração argila dos Latossolos brasileiros. A cristalinidade desses minerais interfere nos atributos físicos do solo, que são dependentes do relevo. Este trabalho teve como objetivo correlacionar espacialmente a estabilidade e o diâmetro médio geométrico dos agregados com os atributos cristalográficos dos minerais da fração argila de um Latossolo Vermelho eutroférrico sob cultivo de cana-de-açúcar com colheita no sistema cana crua. As amostras de solo foram coletadas nos pontos de cruzamento de uma malha, georreferenciados, com intervalos regulares de $10 \mathrm{~m}$ e dimensão de $100 \times 100 \mathrm{~m}$, na profundidade de $0,2-$ $0,4 \mathrm{~m}$, totalizando 119 pontos, em uma área de $1 \mathrm{ha}$, onde as variações da forma do relevo foram mapeadas. Foram determinados o diâmetro médio geométrico e a percentagem de agregados retidos nas classes $>2$ e $<1 \mathrm{~mm}$ das amostras, e a mineralogia da fração argila foi caracterizada por difratometria de raios X. Esses resultados foram submetidos à análise estatística e geoestatística. Buscando correlacionar a estabilidade e as diferentes classes de tamanho dos agregados com
\end{abstract}

\footnotetext{
(1) Recebido para publicação em dezembro de 2007 e aprovado em novembro de 2008.

${ }^{(2)}$ Mestranda do Programa de Pós-Graduação em Agronomia (Produção Vegetal) da FCAV/Unesp, Campus de Jaboticabal. Via de acesso Prof. Paulo Donato Castellani s/nº, CEP 14870-900, Jaboticabal (SP). Bolsista MSc. FAPESP. E-mail: li_arantes@yahoo.com.br

(3) Professor Dr. do Departamento de Solos e Adubos, FCAV/Unesp. E-mail: marques@fcav.unesp.br

(4) Professor Dr. do Departamento de Ciências Exatas, FCAV/Unesp. E-mail: genertp@fcav.unesp.br

(5) Aluna de graduação em Agronomia; Bolsista IC FAPESP; Departamento de Solos e Adubos, FCAV/UNESP. E-mail: renata_horvat@yahoo.com.br
} 
a cristalinidade dos minerais da fração argila do solo, foram realizados semivariogramas cruzados. Observou-se que goethitas de menor grau de cristalinidade se relacionam espacialmente com agregados de maior diâmetro médio geométrico e tamanho dos agregados e que os atributos estudados e suas relações são dependentes das formas do relevo.

Termos de indexação: mineralogia, difração de raios $\mathrm{X}$, geoestatística, semivariograma cruzado.

\title{
SUMMARY: SPATIAL VARIABILITY OF MINERALOGICAL ATTRIBUTES OF AN OXISOL UNDER DIFFERENT RELIEF FORMS. II - CORRELATION BETWEENMINERALOGYAND AGGREGATION
}

\begin{abstract}
Kaolinite, gibbsite, goethite and hematite are the main clay fraction components in Brazilian Oxisols. The abundance of these mineral influence the physical-chemical properties of such soils, which is thought to depend on the relief. This study aimed to characterize the spatial dependence of the crystallinity of kaolinite, gibbsite, goethite and hematite and aggregates of an Oxisol under sugar cane and to assess the spatial correlation between the attributes. In a grid with regular $10 \mathrm{~m}$ intervals 119 samples were collected from a depth of $0.2-0.4 \mathrm{~m}$. in an area of $1 \mathrm{ha}$, where the relief variations were mapped. The mean geometric diameter and the percentage of aggregates in the classes $>2$ and $<1 \mathrm{~mm}$ of the samples, and mineralogy of the clay fraction was characterized by $X$ ray diffraction These results were analyzed statistically and geostatistically. Cross semivariograms were established to correlate stability and the different aggregate size classes with the crystallinity of soil clay minerals. It was observed that the goethites with lowest crystallinity are related to the largest mean geometric diameter and size of aggregate, and that the attributes studied and their relations depend on the relief forms.
\end{abstract}

Index terms: mineralogy, $X$ ray diffraction, geostatistics, cross semivariogram.

\section{INTRODUÇÃO}

Os Latossolos apresentam características físicas que favorecem sobremaneira o seu uso (Oliveira et al., 2004). Contudo, quando submetidos ao uso agrícola intenso, podem sofrer degradação da estrutura e alteração no tamanho dos agregados (Carpenedo \& Mielniczuk, 1990), reduzindo sua estabilidade (Nóbrega et al., 2001). Agregados são os aglomerados de partículas desde o tamanho de argila até maiores que areia. Cada tipo de agregado possui uma gênese própria refletida em seu tamanho, forma, composição e estabilidade (Azevedo \& Bonumá, 2004).

A participação quantitativa dos minerais da fração argila no comportamento dos atributos físicos dos solos é classicamente apresentada pela literatura (McIntyre, 1956; Arca \& Weed, 1966; Schahabi \& Schwertmann, 1970; Goldberg, 1989) e amplamente discutida por autores nacionais (Schwertmann \& Kämpf, 1985; Mesquita Filho \& Torrent, 1993; Pinheiro-Dick \& Schwertmann, 1995; Ferreira et al., 1999a,b; Pedrotti et al., 2003; Guidin et al., 2006a,b). No entanto, alguns aspectos desses estudos ainda são iniciais e carecem de melhor entendimento. Guidin et al. (2006b) salientam que ainda há demanda de esclarecimentos sobre a influência dos atributos cristalográficos dos minerais da fração argila sobre a formação de agregados e definição da porosidade do solo. Por outro lado, estudos indicam a dependência dos atributos físicos e mineralógicos nas diferentes formas do relevo (Souza et al., 2004; Cunha et al., 2005).

Entretanto, Guidin et al. (2006a,b) concluíram que as correlações entre os atributos físicos e os teores de caulinita, gibbsita, hematita e goethita na fração argila não foram significativas para o Latossolo Vermelho distroférrico (LVdf). Esse comportamento foi atribuído à maior homogeneidade das características mineralógicas dessa classe de solo ao longo da toposseqüência estudada. Os autores salientam que, aparentemente, os óxidos de $\mathrm{Fe}$ e $\mathrm{Al}$ de baixa cristalinidade foram mais importantes no incremento da macroporosidade e porosidade total dos horizontes Bw1 e Bw2 do LVdf. Nesses estudos, a cristalinidade dos minerais da fração argila não afetaram, de forma expressiva, os atributos físicos do LVdf e do Latossolo Bruno ácrico (LBw).

Alguns autores consideram a participação de óxidos e hidróxidos de ferro e alumínio, além da caulinita, 
como fator relevante para a presença de agregados com menos de $2 \mathrm{~mm}$ e de alta estabilidade nos Latossolos (Curi \& Franzmeier, 1987; Barberis et al., 1991; Ferreira et al., 1999a; Buol \& Eswaran, 2000), embora não haja consenso sobre os mecanismos envolvidos (Azevedo \& Bonumá, 2004). Contudo, a importância desses minerais para manter as partículas do solo floculadas é amplamente relatada por Ghidin et al. (2006b).

O efeito positivo dos minerais de baixa cristalinidade sobre a agregação do solo foi observado por Pinheiro-Dick \& Schwertmann (1995) e Pedrotti et al. (2003). Esses minerais apresentam alta superfície específica e alta densidade de carga, o que lhes confere grande influência sobre as propriedades físico-químicas do solo, conforme Ghidin et al. (2006b). Esses autores verificaram, em contrapartida, o efeito negativo dos teores dos óxidos de Fe de baixa cristalinidade e, ou, mais cristalinos nos valores do diâmetro médio geométrico (DMG), concordando com os efeitos desses minerais sobre a porosidade dos solos (maiores valores do DMG estão associados a solos com menor volume de macroporos e de porosidade total).

Entretanto, acredita-se que os óxidos pouco cristalinos são os mais eficientes na agregação, devido à sua ação cimentante (Azevedo \& Bonumá, 2004). Essa premissa foi verificada nos estudos sobre agregação em Alfisols e Inceptisols realizados por Barberis et al. (1991). A ação agregante dos óxidos é potencializada por suas ligações com a caulinita, intermediadas por Si (Cambier \& Picot, 1988). O papel dos hidróxidos de Al também é aquilatado pela literatura. Solos com alta razão gibbsita/(gibbsita + caulinita) do Brasil central apresentaram agregados mais estáveis (Pinheiro-Dick \& Schwertmann, 1996). Resultados semelhantes foram encontrados por Vitorino et al. (2003) em estudos enfocando a estabilidade dos agregados do tamanho de silte.

Faccin (1995) observaram que as formas cristalinas de óxidos de $\mathrm{Fe}$ e de $\mathrm{Al}$ não influenciaram a estabilidade dos agregados. Entretanto, teores de hidróxidos de Al correlacionaram-se positivamente com o DMG dos agregados, possivelmente por seu efeito cimentante. Estudos realizados por Ferreira et al. (1999b) demonstraram que Latossolos cauliníticos se apresentam mais compactados devido à sua configuração determinada pela estrutura em blocos, fazendo com que esses solos se apresentem menos permeáveis, com menor estabilidade de agregados em água e maior tendência à erosão laminar.

Os solos apresentam variabilidade ao longo da paisagem em virtude da intensidade de fatores e processos de formação do solo. Quanto maior a variação desses fatores, principalmente a do material de origem e relevo, maior será a heterogeneidade dos solos (Souza et al., 2006). Estudando as relações solopaisagem na região de Monte Alto (SP), Marques Júnior (1995) encontrou variabilidade dos atributos do solo nas diferentes superfícies geomórficas. Cunha et al. (2005), estudando diferentes superfícies geomórficas em Jaboticabal (SP), encontraram maior variabilidade espacial dos atributos mineralógicos na superfície mais nova em relação à mais antiga, evidenciando que solos intemperizados, como os Latossolos, em superfícies mais recentes são mineralogicamente menos homogêneos do que aqueles situados em superfícies mais antigas (normalmente mais planas). Esses autores encontraram variabilidade espacial para $\mathrm{Ct}$ e $\mathrm{Gb}$ relacionada à variação dos teores de Fe em função do relevo da área estudada. Segundo Pocay (2000), o comportamento de atributos físicos, químicos e mineralógicos em diferentes toposseqüências informa sobre o padrão de distribuição espacial diferenciado nesses ambientes, principalmente quando associado aos estudos pedológicos e geomórficos.

Assim, o objetivo deste trabalho foi correlacionar espacialmente os atributos cristalográficos dos minerais da fração argila do solo com diversas classes de agregados sob variações do relevo.

\section{MATERIAL E MÉTODOS}

\section{Localização, caracterização do meio físico e critério de amostragem}

A área de estudo localiza-se no nordeste do Estado de São Paulo, no município de Guariba (SP). O clima da região, segundo a classificação de Köppen, é do tipo mesotérmico com inverno seco (Cwa), com precipitação média de $1.400 \mathrm{~mm}$, com chuvas concentradas no período de novembro a fevereiro. A vegetação natural é constituída por floresta tropical subcaducifólia. O solo da área foi classificado como Latossolo Vermelho eutroférrico textura muito argilosa (LVef), segundo critérios da Embrapa (2006), sob cultivo de cana-deaçúcar, com colheita sob sistema cana crua. As amostras de solo para avaliação dos agregados foram coletadas nos pontos de cruzamento de uma malha, georreferenciados com intervalos regulares de $10 \mathrm{~m}$, na profundidade de 0,2-0,4 m, com dimensão de 100 x 100 m, totalizando 119 pontos, em uma área de 1 ha. A caracterização dos minerais da fração argila foi realizada e pode ser encontrada com detalhes em Camargo et al. (2008), assim como o Modelo Digital de Elevação (MDE) da área de estudo que foi utilizado para identificação das formas do relevo. Por meio do MDE, foram identificados dois compartimentos (I e II): no I, observa-se uma área linear com declive menos acentuado; e no II, uma área com forma côncavoconvexa.

\section{Métodos de laboratório}

A separação e a estabilidade dos agregados foram determinadas pelo método descrito por Kemper \& Chepil (1965), com modificações, nas seguintes classes de diâmetro: > 2,0; 2,0-1,0; 1,0-0,5; 0,5-0,25; 0,25- 
0,125 e 0,125-0,105 mm. As classes de agregados utilizadas neste estudo foram: $>2,0$ e $<1,0 \mathrm{~mm}$. Uma vez secas ao ar, as amostras com estrutura natural foram passadas em peneiras de malhas de 9,52 e $4,76 \mathrm{~mm}$. Os agregados retidos na peneira de 4,76 foram submetidos ao pré-umedecimento lento por meio de atomização com água destilada e, posteriormente, foram agitados, por $15 \mathrm{~min}$, em jogo de peneira com malhas de 2,$0 ; 1,0 ; 0,5 ; 0,25 ; 0,125 ;$ e 0,105 , dentro de um recipiente que continha água. A massa do material retido em cada peneira foi colocada em estufa a $105^{\circ} \mathrm{C}$. Mediante os resultados obtidos, foi calculado o diâmetro médio geométrico (DMG), que será adotado como índice de estabilidade, de acordo com Mazurack (1950).

\section{Análises estatística e geoestatística}

Os dados foram submetidos à análise estatística descritiva, calculando-se a média, a mediana, a variância, o desvio-padrão, a assimetria, a curtose, o máximo, o mínimo, o coeficiente de variação e o tipo de distribuição. Na análise estatística dos dados, foi utilizado o programa Minitab (2000).

Para caracterização do padrão da variabilidade espacial, foi empregada a análise geoestatística (Vieira, 2000). Foram construídos semivariogramas com base no cálculo da semivariância $\gamma(\mathrm{h})$, por meio da seguinte equação:

$$
\hat{\gamma}(h)=\frac{1}{2 N(h)} \sum_{i=1}^{N(h)}\left[Z\left(x_{i}\right)-Z\left(x_{i}+h\right)\right]^{2}
$$

em que: $\hat{\gamma}(h)$ é a semivariância estimada a partir dos dados experimentais; $Z$ são os valores medidos nos pontos $\mathrm{x}_{\mathrm{i}}$ e $\mathrm{x}_{\mathrm{i}}+\mathrm{h}$; e $\mathrm{N}(\mathrm{h})$ é o número de pares de valores medidos separados por uma distância h. Os modelos foram ajustados por meio do programa GS+ (Robertson, 1998) e, em caso de dúvida entre mais de um modelo para o mesmo semivariograma, foi utilizada a técnica de validação cruzada. Observada a dependência espacial entre as amostras, definida pelo semivariograma, é possível estimar valores em que a variável não foi medida, fazendo uso da krigagem, segundo Vieira et al. (2002).

No sentido de averiguar a correlação entre os atributos em estudo, foram construídos semivariogramas cruzados, que podem assumir valores tanto positivos, indicando que o aumento em um dos atributos é acompanhado pelo aumento do outro, como negativos, indicando que, quando ocorre o aumento de um dos atributos, ocorre o decréscimo do outro, segundo Bhatti et al. (1991) citados por Mata (1997). A confecção dos mapas de krigagem foi feita utilizando-se o programa Surfer (1999).

\section{RESULTADOS E DISCUSSÃO}

De todos os atributos estudados, apenas a percentagem de agregados na classe $>2 \mathrm{~mm}$ não apresentou distribuição normal (Quadro 1). Todavia, todos os atributos possuem distribuições simétricas, devido aos valores de assimetria próximos de zero. Segundo Cressie (1991), em se tratando de dados obtidos na natureza, o ajuste de uma distribuição teórica é apenas aproximado, não sendo necessária, portanto, a normalidade dos dados para as análises geoestatísticas, e sim que a distribuição dos dados não tenha caudas muito alongadas.

Adotando-se a classificação para o coeficiente de variação (CV) proposta por Warrick \& Nielsen (1980), os agregados $<1 \mathrm{~mm}$ apresentaram valor alto (> $24 \%$ ). O valor de CV para os agregados $>2 \mathrm{~mm}$ foi classificado como médio (entre 12 e $24 \%$ ), e para DMG, baixo (<12\%). A medida estatística CV permite comparar a variabilidade entre amostras de variáveis com unidades diferentes, porém não permite analisar a variabilidade espacial dos atributos do solo nem seu padrão espacial. Nesse sentido, a variação dos atributos observada neste estudo justifica a análise da dependência espacial para melhor entendimento do padrão de ocorrência desses atributos.

No quadro 2 são apresentados os coeficientes de correlação simples entre os atributos mineralógicos citados por Camargo et al. (2008) e os atributos DMG e agregados das classes $>2,0$ e $<1,0 \mathrm{~mm}$. Observase que houve correlação positiva significativa entre largura à meia altura (LMA) da Gt e proporção de agregados $>2,0 \mathrm{~mm}$.

Para avaliação da variabilidade e dependência espacial dos atributos estudados, foram estimados semivariogramas experimentais isotrópicos (Figura 1). Todos os atributos apresentaram dependência espacial, e o modelo que melhor se ajustou aos semivariogramas experimentais foi o exponencial. A relação $\mathrm{C}_{0} /\left(\mathrm{C}_{0}+\mathrm{C}_{1}\right)$ em percentagem do atributo DMG e agregados $>2 \mathrm{~mm}$ indica grau de dependência espacial forte $\left[\mathrm{C}_{0} /\right.$ $\left(\mathrm{C}_{0}+\mathrm{C}_{1)}<25 \%\right.$ ]; para o atributo agregados $<1 \mathrm{~mm}$,

Quadro 1. Estatísticas descritivas do diâmetro médio geométrico (DMG) e das classes de agregados: $>2,0$ e $<1,0 \mathrm{~mm}$ de 119 amostras coletadas na profundidade de $0,2-0,4 \mathrm{~m}$

\begin{tabular}{lccc}
\hline Parâmetro & DMG $^{(1)}$ & $\mathbf{2} \mathbf{~ m m}$ & $<\mathbf{1 ~} \mathbf{m m}$ \\
\hline Mm & \multicolumn{2}{c}{$\%$} & \\
Média & 1,47 & 69,80 & 11,48 \\
Variância & 0,009 & 111,79 & 11,43 \\
Assimetria & 0,19 & $-0,85$ & 0,87 \\
Curtose & 2,59 & 1,44 & 0,92 \\
Desvio-padrão & 0,097 & 10,57 & 3,38 \\
CV $^{(2)}$ (\%) & 6,53 & 15,15 & 29,44 \\
Valor p $^{(3)}$ & 0,036 & 0,017 & 0,006
\end{tabular}

(1) Diâmetro médio geométrico. ${ }^{(2)}$ Coeficiente de variação. ${ }^{(3)} p$ significativo a $5 \%$ (distribuição normal pelo teste de AndersonDarling) 
Quadro 2. Correlação entre os atributos mineralógicos dos minerais da fração argila e diâmetro médio geométrico (DMG) e as classes de agregados: $>2,0$ e $<1,0 \mathrm{~mm}$ de 119 amostras coletadas na profundidade de $0,2-0,4 \mathrm{~m}$

\begin{tabular}{|c|c|c|c|c|}
\hline & & DMG & $>2 \mathrm{~mm}$ & $<1 \mathrm{~mm}$ \\
\hline & & $\mathrm{mm}$ & 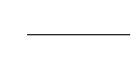 & $\%$ \\
\hline $\mathrm{LMA}^{(1)}$ & $\begin{array}{l}\mathrm{Hm} \\
\mathrm{Gt} \\
\mathrm{Ct} \\
\mathrm{Gb}\end{array}$ & $\begin{array}{r}0,051^{\mathrm{ns}} \\
0,165^{\mathrm{ns}} \\
-0,069^{\mathrm{ns}} \\
0,170^{\mathrm{ns}}\end{array}$ & $\begin{array}{r}0,051^{\mathrm{ns}} \\
0,274^{* *} \\
-0,062^{\mathrm{ns}} \\
0,140^{\mathrm{ns}}\end{array}$ & $\begin{array}{r}-0,033^{\mathrm{ns}} \\
-0,172^{\mathrm{ns}} \\
0,103^{\mathrm{ns}} \\
-0,023^{\mathrm{ns}}\end{array}$ \\
\hline $\mathrm{DMC}^{(2)}$ & $\begin{array}{l}\mathrm{Hm} \\
\mathrm{Gt} \\
\mathrm{Ct} \\
\mathrm{Gb}\end{array}$ & $\begin{array}{r}-0,051^{\mathrm{ns}} \\
-0,074^{\mathrm{ns}} \\
0,038^{\mathrm{ns}} \\
0,128^{\mathrm{ns}}\end{array}$ & $\begin{array}{r}-0,035^{\mathrm{ns}} \\
-0,163^{\mathrm{ns}} \\
0,074^{\mathrm{ns}} \\
0,117^{\mathrm{ns}}\end{array}$ & $\begin{array}{r}0,017^{\mathrm{ns}} \\
0,092^{\mathrm{ns}} \\
-0,064^{\mathrm{ns}} \\
0,026^{\mathrm{ns}}\end{array}$ \\
\hline
\end{tabular}

(1) Largura à meia altura ( $\left.{ }^{\circ} 2 \theta\right) .{ }^{(2)}$ Diâmetro médio do cristal (nm). ${ }^{* *}$ significativo a $1 \%$; ${ }^{\text {ns }}$ não-significativo; Hm: hematita; Gt: goethita; Ct: caulinita, e Gb: gibbsita.

(a) DMG, $\mathbf{m m}$
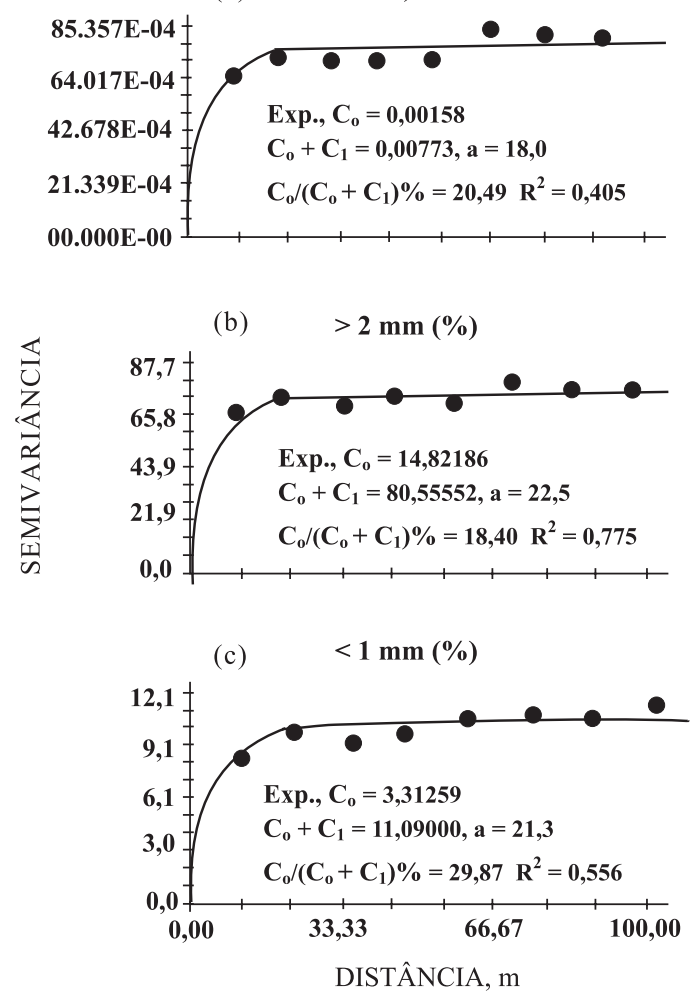

Figura 1. Semivariogramas dos atributos estudados: (a) diâmetro médio geométrico (DMG) (b) agregados da classe $>2,0 \mathrm{~mm}$; (c) agregados da classe $<1,0 \mathrm{~mm}$; Exp.: exponencial; $\mathrm{C}_{\mathrm{o}}$ : efeito pepita; $C_{1}$ : patamar; a: alcance; $R^{2}$ : coeficiente de determinação.

indica grau de dependência espacial moderado $\left[\mathrm{C}_{0} /\right.$ $\left(\mathrm{C}_{0}+\mathrm{C}_{1}\right)$ entre 25 e $75 \%$ ], segundo a classificação proposta por Cambardella et al. (1994).
Carvalho et al. (2004) também encontraram variabilidade espacial nas diferentes classes de agregados na mesma classe de solo deste estudo, e concluíram que a distribuição espacial dos agregados não foi ao acaso, e sim resultante de processos naturais e de práticas culturais. A avaliação da variabilidade espacial dos atributos estudados mostra que, mesmo na classe dos Latossolos sob cultivo de cana-de-açúcar por longo tempo, pode-se registrar variabilidade espacial da estabilidade e tamanho dos agregados.

Como todos os atributos apresentaram estrutura espacial, os parâmetros dos semivariogramas ajustados foram utilizados para estimar valores em locais não amostrados por meio da krigagem (Figura 2).

Nos mapas de distribuição espacial obtidos, com exceção do DMG, observa-se que a distribuição dos agregados das classes $>2,0$ e $<1,0 \mathrm{~mm}$ coincide com a divisão dos dois compartimentos da área, semelhante ao que ocorre com os atributos mineralógicos estudados na área por Camargo et al. (2008), e que no compartimento I (áreas com forma linear) se encontram os maiores valores das percentagens de

\section{(a)}

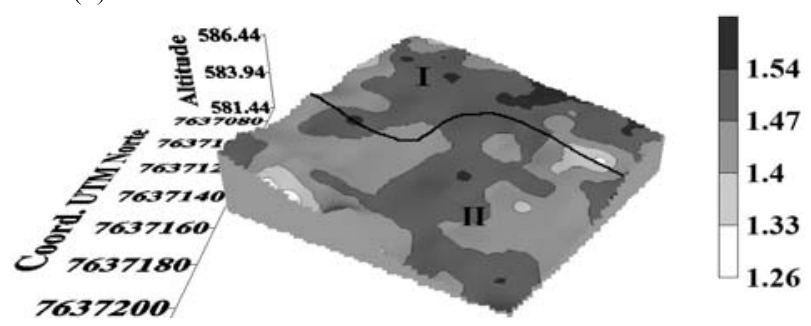

$>2 \mathrm{~mm}(\%)$

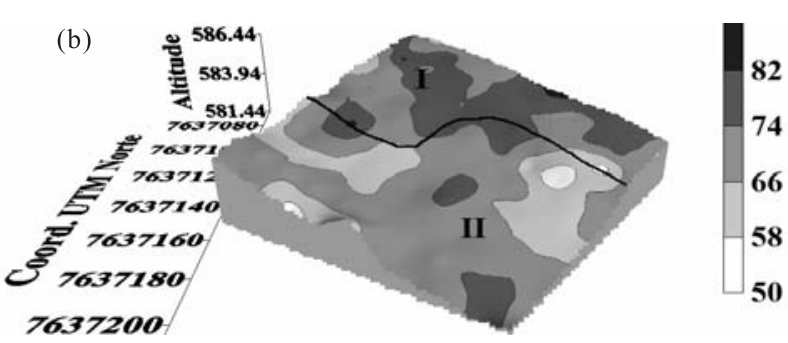

$<1 \mathrm{~mm}(\%)$

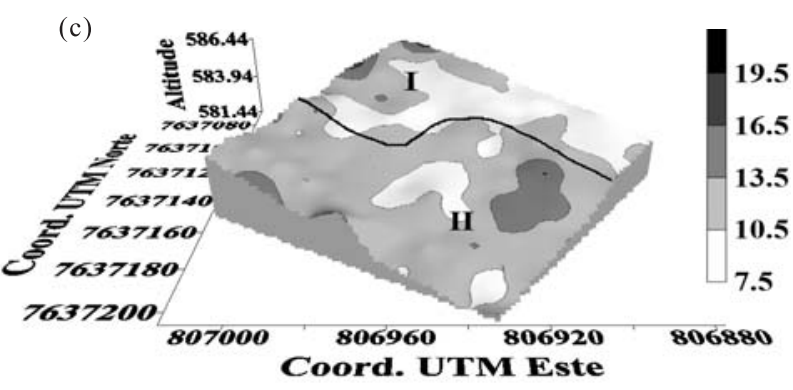

Figura 2. Mapas do padrão espacial do diâmetro médio geométrico (DMG) e das classes de agregados: $>2,0 \mathrm{e}<1,0 \mathrm{~mm}$. 
agregados $>2 \mathrm{~mm}$ e os menores valores das percentagens dos agregados $<1 \mathrm{~mm}$.

Souza et al. (2004) também observaram a variabilidade espacial da estabilidade do agregado e das diferentes classes de tamanho dos agregados de um Latossolo e atribuíram-na às diferentes formas do relevo.

Buscando correlacionar espacialmente os atributos mineralógicos, a estabilidade dos agregados e tamanhos deles, foram realizados os semivariogramas cruzados entre esses atributos (Figuras 3, 4 e 5). As correlações espaciais positivas encontradas foram entre os seguintes atributos: DMG e LMA da Gt; agregados $>2,0 \mathrm{~mm}$ e LMA da Gt; DMG e DMC da Ct; e agregados $>2,0 \mathrm{~mm}$ e DMC da Ct. As correlações espaciais negativas foram encontradas entre: DMG e DMC da Gt; agregados > 2,0 mm e DMC da Gt;

(a) DMG em função LMA da Hm

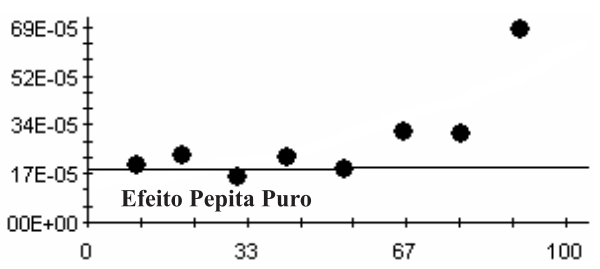

(c) DMG em função DMC da Hm

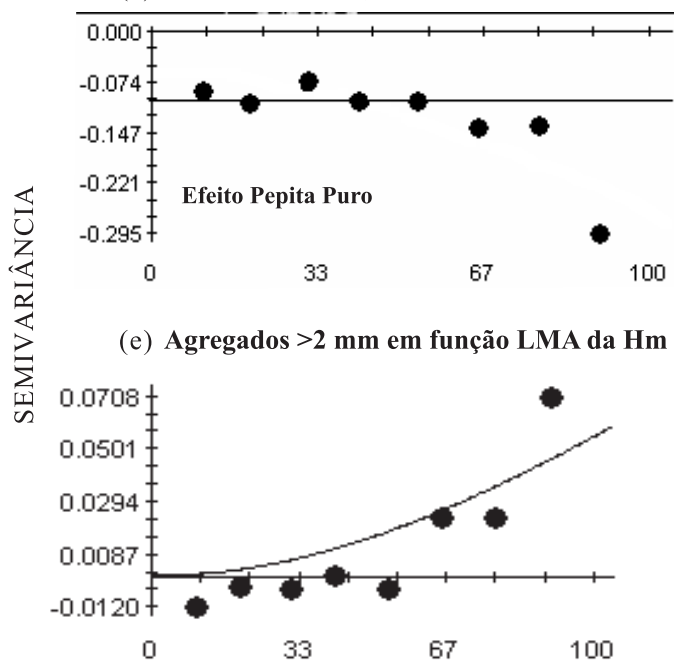

(g) Agregados $>2$ mm em função DMC da Hm

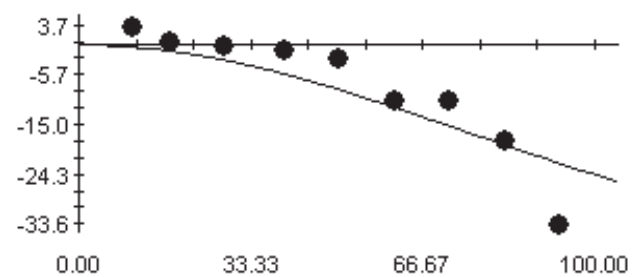

DISTÂNCIA, m agregados < 1,0 mm e LMA da Gt. Nos semivariogramas cruzados apresentados nas figuras 3e,g, 4a,c,d,e,f, $5 \mathrm{a}, \mathrm{e}, \mathrm{f}, \mathrm{h}$ as correlações espaciais mostram-se indefinidas.

Entre as correlações espaciais, é possível observar que o grau de cristalinidade da Gt se relaciona com agregados do solo. Nota-se que o DMG e os agregados $>$ 2,0 $\mathrm{mm}$ aumentam com o aumento da LMA da Gt, e os agregados $<1,0 \mathrm{~mm}$ diminuem com o aumento da LMA da Gt, ou seja, goethitas de menor cristalinidade, evidenciada pela maior LMA e menor DMC (Fitspatrick \& Schwertmann, 1982), relacionamse positivamente com a estabilidade e os agregados de maior tamanho e negativamente com os agregados de menor tamanho. Esses resultados podem ser explicados pela maior superfície específica das goethitas de menor grau de cristalinidade (Strauss et

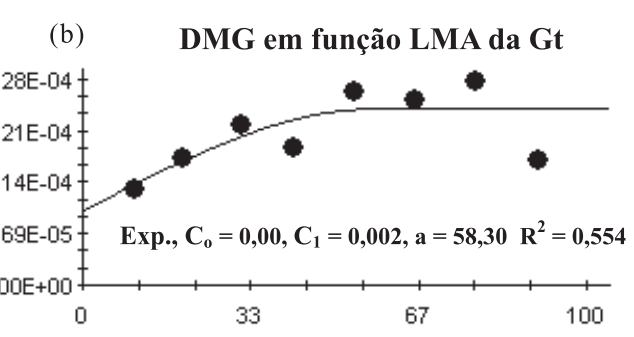

(d) DMG em função DMC da Gt

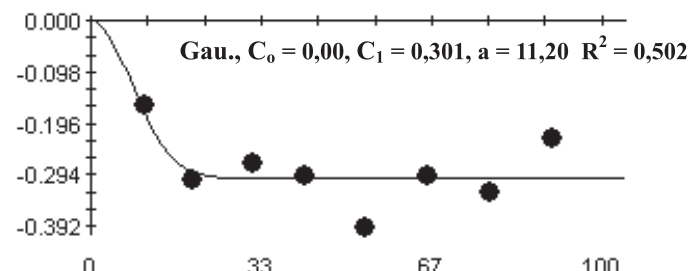

(f) Agregados $>\mathbf{2} \mathbf{~ m m}$ em função LMA da Gt

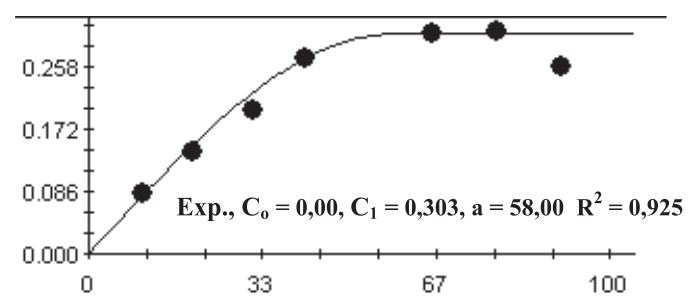

(h) Agregados >2 mm em função DMC da Gt

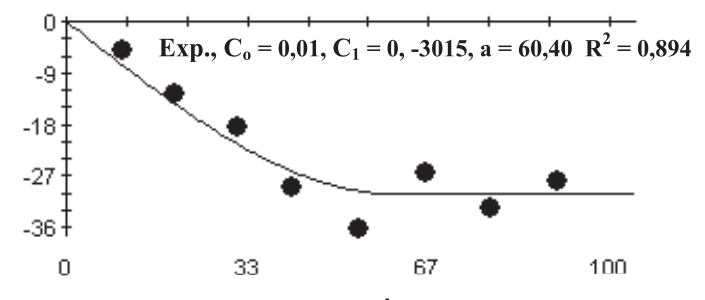

DISTÂNCIA, m

Figura 3. Semivariogramas cruzados dos atributos estudados. Gau.: gaussiano, Esf.: esférico; $C_{0}$ : efeito pepita; $\mathrm{C}_{1}$ : patamar; a: alcance $(\mathrm{m}) ; \mathrm{R}^{2}$ : coeficiente de determinação. 
(a) Agregados $<1 \mathrm{~mm}$ em função LMA da $\mathrm{Hm}$

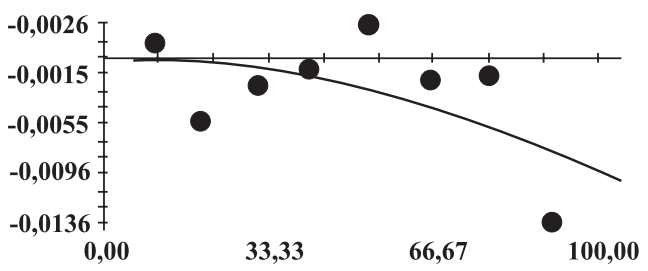

(c) Agregados $<1 \mathrm{~mm}$ em função DMG da $\mathrm{Hm}$

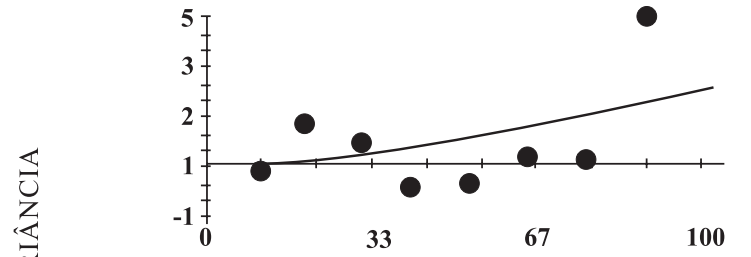

(e) DMG em função LMA da Ct

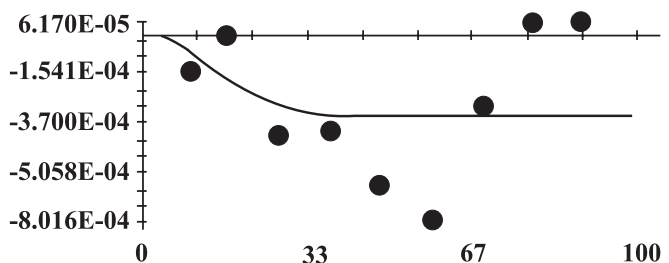

(g) DMG em função DMC da Ct

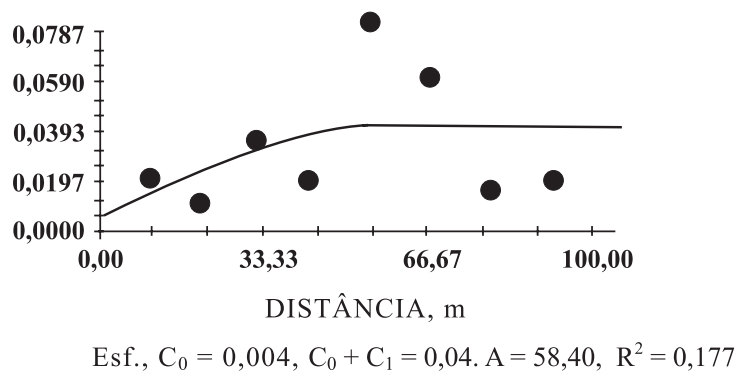

(b) Agregados $<1$ mm em função LMA da Gt

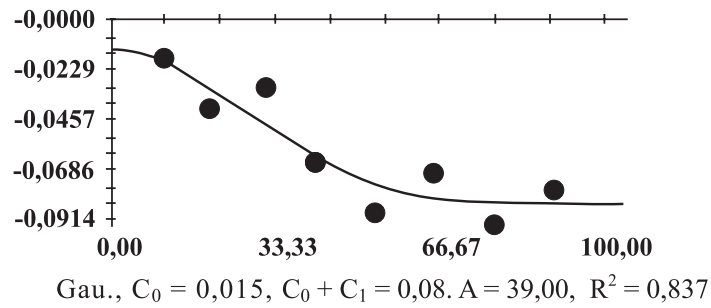

(d) Agregados $<1$ mm em função DMG da Gt

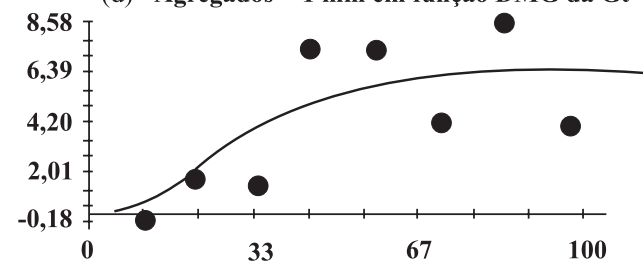

(f) DMG em função LMA da Gb

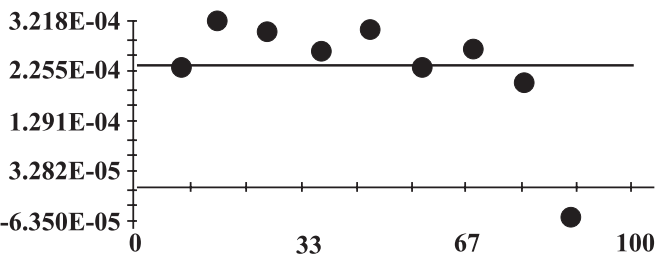

(h) DMG em função DMC da Gb

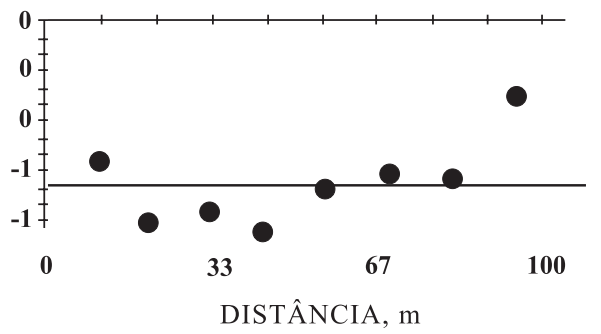

Efeito Pepita Puro

Figura 4. Semivariogramas cruzados dos atributos estudados. Gau.: gaussiano; Esf.: esférico; $\mathrm{C}_{\mathrm{o}}$ : efeito pepita; $C_{1}$ : patamar; a: alcance $(m) ; R^{2}$ : coeficiente de determinação.

al., 1997) e, conseqüentemente, maior densidade de carga, contribuindo para a agregação do solo (Cornell \& Schwertmann, 1996). Esse resultado também foi observado por Pinheiro-Dick \& Schwertmann (1995) e corroboram os estudos de Azevedo \& Bonumá (2004).

Não houve, entretanto, correlação espacial entre os atributos cristalográficos da $\mathrm{Hm}$ e as classes de agregados, revelando que a cristalinidade desse mineral está menos relacionada com a agregação do solo estudado. Barberis et al. (1991) também não encontraram correlação entre Hm e a agregação do solo e relatam que pedoambientes favoráveis à formação de hematitas agem em sentido oposto na agregação.

O DMC da Ct correlacionou-se positivamente com o DMG e com os agregados $>2 \mathrm{~mm}$. Vitorino et al. (2003) encontraram correlação direta com teores de
Gb e inversa com teores de Ct na formação e estabilização de agregados do solo, destacando a importância do $\mathrm{Al}$ na resistência à desagregação.

Ferreira et al. (1999a), estudando Latossolos da região Sudeste, observaram a correlação entre a estabilidade do agregado e a mineralogia da fração argila e concluíram que a estabilidade do agregado é diretamente influenciada pelos teores de Gb, porém não fizeram inferência sobre a cristalinidade desse mineral. Já nos estudos de Pedrotti et al. (2003) não foram encontradas correlações significativas entre os teores de $\mathrm{Ct}$ e $\mathrm{Gb}$ e estabilidade do agregado. Assim, ressalta-se que o conhecimento da cristalinidade dos minerais da fração argila do solo e a relação desta com as formas do relevo permitem inferir a estabilidade e a distribuição dos diferentes tamanhos dos agregados na paisagem. 
(a) Agregados $>2 \mathrm{~mm}$ em função LMA da Ct

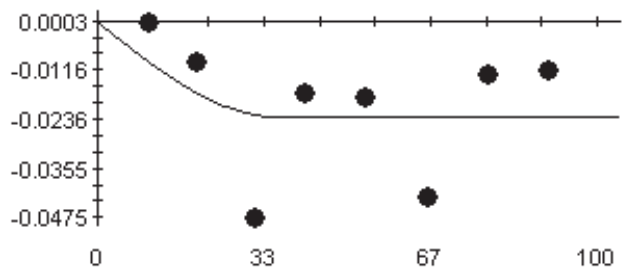

(c) Agregados $>2 \mathrm{~mm}$ em função DMC da Ct

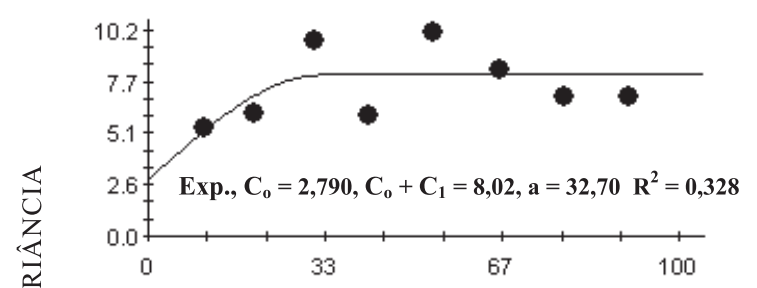

(e) Agregados $<1 \mathrm{~mm}$ em função LMA da Ct

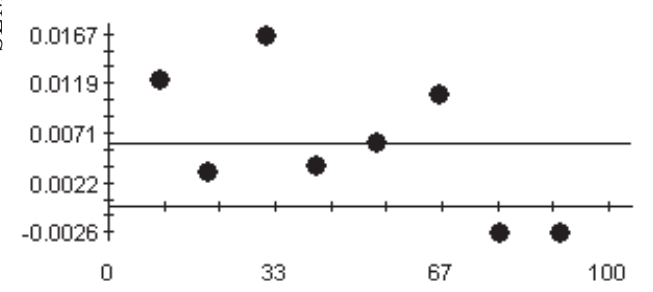

(g) Agregados $<1$ mm em função DMC da Ct

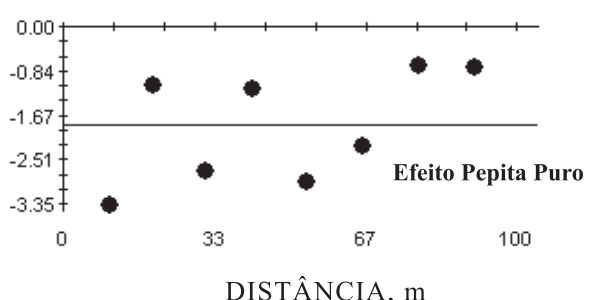

(b) Agregados $>\mathbf{2} \mathbf{~ m m}$ em função LMA da Gb

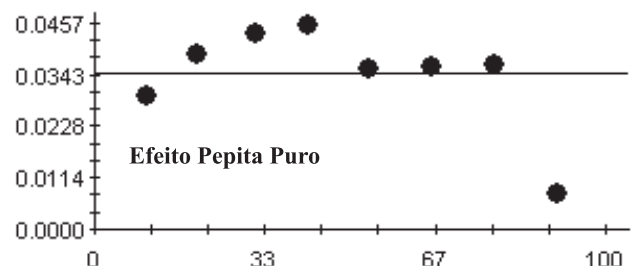

(d) Agregados $>2$ mm em função DMC da Gb

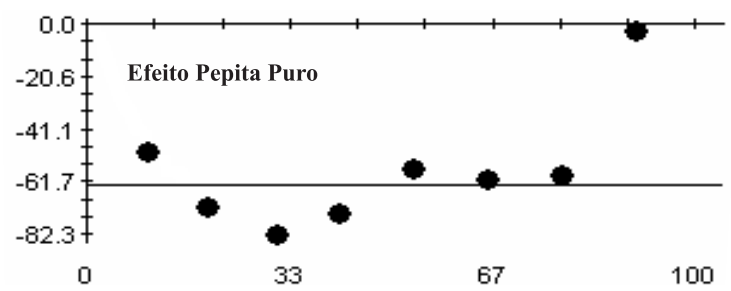

(f) Agregados $<1 \mathrm{~mm}$ em função LMA da Gb

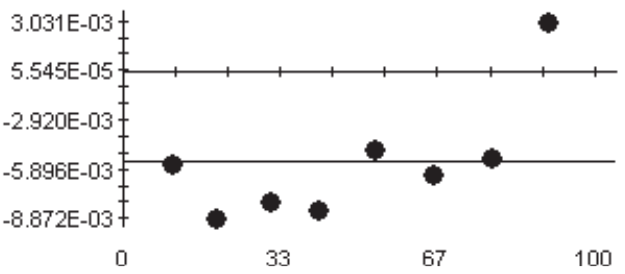

(h) Agregados $<1 \mathrm{~mm}$ em função DMC da Gb

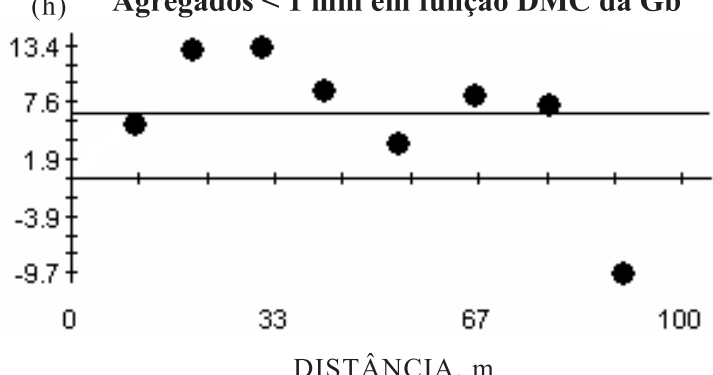

Figura 5. Semivariogramas cruzados dos atributos estudados. Esf.: esférico; $C_{0}$ : efeito pepita; $C_{1}$ : patamar; a: alcance $(m) ; R^{2}$ : coeficiente de determinação.

\section{CONCLUSÕES}

1. O diâmetro médio geométrico e as classes de agregados $>2,0$ e $<1,0 \mathrm{~mm}$ apresentaram dependência espacial.

2. No compartimento I (linear) encontram-se os maiores valores das percentagens de agregados da classe $>2 \mathrm{~mm}$ e os menores da classe $<1 \mathrm{~mm}$.

3. Goethita e caulinita de menor e maior grau de cristalinidade respectivamente relacionam-se positivamente com agregados mais estáveis e de maior tamanho. Não houve relação entre a hematita e os atributos referentes aos agregados.

\section{AGRADECIMENTOS}

À Usina São Martinho, pela concessão da área de estudo.

À Fundação de Amparo à Pesquisa do Estado de São Paulo (FAPESP), pela concessão de bolsa à primeira e quarta autoras.

\section{LITERATURA CITADA}

ARCA, M.N. \& WEED, S.B. Soil aggregation and porosity in relation to contents of free iron oxide and clay. Soil Sci., 109:282-288, 1966. 
AZEVEDO, A.C. \& BONUMÁ, A.S. Partículas coloidais, dispersão e agregação em Latossolos. Ci. Rural, 34:609$617,2004$.

BARBERIS, E. et al. Aggregation of soil particles by iron oxides in various size fractions of soil B horizons. J. Soil Sci., 42:535-542, 1991.

BHATTI, A.U.; MULLA, D.J.; KOEHLER, F.E. \& GURMANI, A.H. Identifying and removing spatial correlation from yield experiments. Soil Sci. Soc. Am. J., 55:1523-1528, 1991.

BUOL, S. W. \& ESWARAN, H. Oxisols. Adv. Agron., 68:151$195,2000$.

CAMARGO, L.A.; MARQUES JR, J.; PEREIRA, G.T. \& HORVAT, R.A. Variabilidade espacial de atributos mineralógicos de um Latossolo sob diferentes formas do relevo. I - Mineralogia da fração argila. R. Bras. Ci. Solo, 32:2269-2277, 2008.

CAMBARDELLA, C.A.; MOORMAN, T.B.; NOVAK, J.M.; PARKIN, T.B.; KARLEN, D.L; TURCO R.F. \& KONOPKA, A.E. Field scale variability of soil properties in central Iowa soils. Soil Sci. Soc. Am. J., 58:1501-1511, 1994.

CAMBIER, P. \& PICOT, C. Nature des liaisons kaolinite-oxyde de fer au sein des microagregats d'un sol ferralitique. Sci. Sol, 26:233-238, 1988.

CARPENEDO, V. \& MIELNICZUK, J. Estado de agregação e qualidade de agregados de Latossolos Roxos, submetidos a diferentes sistemas de manejo. R Bras. Ci. Solo, 14:99$105,1990$.

CARVALHO, J.R.P.; DECHEN, S.C.F. \& DUFRANC, G Variabilidade espacial da agregação do solo avaliada pela geometria fractal e geoestatística. R. Bras. Ci. Solo, 28:19, 2004 .

CORNELL, R.M. \& SCHWERTMANN, U. The iron oxides. Structure, properties, reactions, occurrence and uses, 1996. Weinheim, VHC, 1996. 573p.

CRESSIE, N. Statistics for spatial data. New York, John Wiley, 1991. 900p.

CUNHA, P.; MARQUES JÚNIOR, J.; CURI, N.; PEREIRA, G.T. \& LEPSCH, I.F. Superfícies geomórficas e atributos de Latossolos em uma topossequência arenítico-basáltica da região de Jaboticabal (SP). R. Bras. Ci. Solo, 29:81-90, 2005.

CURI, N. \& FRANZMEIER, D.P. Effect of parent rock on chemical and mineralogical properties of some Oxisols in Brazil. Soil Sci. Soc. Am. J, 51:153-158, 1987.

EMPRESA BRASILEIRA DE PESQUISA AGROPECUÁRIA EMBRAPA. Centro Nacional de Pesquisa de Solos. Sistema brasileiro de classificação de solos. 2.ed. Rio de Janeiro, 2006. 306p.

FACCIN, O.P. Influência das características químicas, físicas e mineralógicas sobre a estabilidade de agregados de diferentes grupamentos de solos. Lavras, Universidade Federal de Lavras 1995. 67p. (Tese de Mestrado)
FERREIRA, M.M.; FERNANDES, B. \& CURI, N. Influência da mineralogia da fração argila nas propriedades físicas de Latossolos da Região Sudeste do Brasil. R. Bras. Ci. Solo, 23:515-524, 1999a.

FERREIRA, M.M.; FERNANDES, B. \& CURI, N. Mineralogia da fração argila e estrutura de Latossolos da Região Sudeste do Brasil. R. Bras. Ci. Solo, 23:507-514, 1999b.

FITZPATRICK, R.W. \& SCHWERTMANN, U. Al-Substituted goethite as indicator of pedogenic and other weathering environments in South Africa. Geoderma, 27:335347,1982 .

GHIDIN, A.A.; MELO, V.F.; LIMA, V.C. \& LIMA, J.M.J.C. Topossequências de Latossolos originados de rochas basálticas no Paraná. I - Mineralogia da fração argila. R. Bras. Ci. Solo, 30:293-306, 2006a.

GHIDIN, A.A.; MELO, V.F.; LIMA, V.C. \& LIMA, J.M.J.C. Topossequências de Latossolos originados de rochas basálticas no Paraná. II - Relação entre mineralogia da fração argila e propriedades físicas dos solos. R. Bras. Ci. Solo, 30:307-319, 2006b.

GOLDBERG, S. Interactions of aluminum and iron oxides and clay minerals and their effect on soil physical properties: A review. Comm. Soil Sci. Plant Anal., 20:11811207. 1989.

KEMPER, W.D. \& CHEPIL, W.S. Size distribution of aggregates. In: BLACK, C.A.; EVANS, D.D.; WHITE, J.L.; ENSMINGER, L.E. \& CLARK, F.E., eds. Methods of soil analysis: Physical and mineralogical properties, including statistics of measurement and sampling. Madison, American Society of Agronomy, 1965. p.499-510.

MARQUES JÚNIOR, J. Distribuição e atributos dos solos em relação à forma e evolução de uma vertente em Monte Alto, SP. Piracicaba, Escola Superior de Agricultura Luiz de Queiroz, 1995. 226p. (Tese de Doutorado)

MATA, J.D.V. Variabilidade espacial de indicadores da compactação de terra roxa estruturada, sob dois sistemas de preparo, cultivada com feijão (Phaseolus vulgaris L.) irrigado. Piracicaba, Escola Superior de Agricultura Luiz de Queiroz, 1997. 73p. (Tese de Doutorado)

MAZURACK, A.P. Effect of gaseous phase on water-stable synthetic aggregates. Soil Sci., 69:135-148, 1950.

McINTYRE, D.S. The effect of free ferric oxide on the structure of some Terra Rossa and Rendzina soils. J. Soil Sci., 7:302306, 1956.

MESQUITA FILHO, M.V. \& TORRENT, J. Phosphate sorption as related to mineralogy of a hydrosequence of soils from Cerrado region (Brazil). Geoderma, 58:107-123, 1993.

MINITAB Release. Making Data analysis Easler: version 13.1, 2000 .

NÓBREGA, J.C.A.; LIMA, J.M.; CURI, N.; SIQUEIRA, J.O. \& MOTTA, P.E.F. Fosfato e micorriza na estabilidade de agregados em amostras de Latossolos cultivados e nãocultivados. Pesq. Agropec. Bras., 36:1425-1435, 2001. 
OLIVEIRA, G.C.; DIAS JUNIOR, M.S.; RESCK, D.V.S. \& CURI, N. Caracterização química e físico-hídrica de um Latossolo Vermelho após vinte anos de manejo e cultivo do solo. R. Bras. Ci. Solo, 28:327-336, 2004.

PEDROTTI, A.; FERREIRA, M.M.; CURI, N.; SILVA, M.L.N.; LIMA, J.M. \& CARVALHO, R. Relação entre atributos físicos , mineralogia da fração argila e formas de alumínio no solo. R. Bras. Ci. Solo, 27:1-9, 2003.

PINHEIRO-DICK, D. \& SCHWERMANN, U. Características químicas, mineralógicas e morfológicas de microagregados de Oxissolos. In: CONGRESSO BRASILEIRO DE CIÊNCIA DO SOLO, 25., Viçosa, MG, 1995. Resumos expandidos. Viçosa, MG, Sociedade Brasileira de Ciência do Solo, 1995. p.262-264.

PINHEIRO-DICK, D. \& SCHWERTMANN, U. Microagregates from Oxisols and Inceptisols: Dispersion through selective dissolution's and physicochemical treatments. Geoderma, 74:49-63, 1996

POCAY, V.G. Relações entre pedoforma e variabilidade espacial de atributos de Latossolos sob cultivo de cana-de-açúcar. Jaboticabal, Universidade Estadual Paulista, 2000. 177p. (Tese de Mestrado)

ROBERTSON, G.P. GS $^{+}$geostatistics for the environmental sciences: GS $^{+}$user' guide. Plainwell, Gamma Design Software, 1998. 152p

SCHWERTMANN, U. \& KÄMPF, N. Properties of goethite and hematite in kaolinitic soils of Southern and Central Brazil. Soil Sci.,139:344-50, 1985.

SCHAHABI, S. \& SCHWERTMANN, U. Der Einfluâ von synthetischen Eisenoxidem auf die Aggregation zweier Lößbödenhorizonte. Z. Pflanzenernähr; Bodenkd., 125:193-204, 1970.
SOUZA, Z.M.; MARQUES JÚNIOR, J. \& PEREIRA, G.T. Variabilidade espacial de atributos físicos do solo em diferentes formas do relevo sob cultivo de cana-de-açúcar. R. Bras. Ci. Solo, 28:937-944, 2004.

SOUZA, Z.M.; MARQUES JUNIOR, J.; PEREIRA, G.T. \& BARBIERI, D.M. Small relief shape variations influence spatial variability of soil chemical attributes. Sci. Agric., 63:161-168, 2006

STRAUSS, R.; BRÜMMER, G.W. \& BARROW, N.J. Effects of crystallinity of goethite: II. Rates of sorption and desorption of phosphate. Eur. J. Soil Sci., 48:101-114, 1997.

SURFER. Surfer 7.0. Contouring and 3D surface mapping for scientist's engineers: User's guide. New York, Golden Software, 1999. 619p.

VIEIRA, S.R. Geoestatística em estudos de variabilidade espacial do solo. In: NOVAIS, R.F.; ALVAREZ V., V.H. \& SCHAEFER, C.E.G.R., eds. Tópicos em ciência do solo. Viçosa, MG, Sociedade Brasileira de Ciência do Solo, 2000. v.1. p.1-53.

VIEIRA, S.R.; MILLETE, J.; TOPP, G.C. \& REYNOLDS, W.D. Handbook for geostatistical analysis of variability in soil and climate data. In: ALVAREZ V., V.H.; SCHAEFER, C.E.G.R.; BARROS, N.F.; MELLO, J.W.V. \& COSTA, L.M., eds. Tópicos em ciência do solo, Viçosa, MG, Sociedade Brasileira de Ciência do Solo, 2002. v.2. p.1-45.

VITORINO, A.C.T.; FERREIRA, M.M.; CURI, N.; LIMA, J.M.; SILVA, M.L.N. \& MOTTA, P.E.F. Mineralogia, química e estabilidade de agregados do tamanho de silte de solos da região Sudeste do Brasil. Pesq. Agropec.Bras., 38:133$141,2003$.

WARRICK, A.W. \& NIELSEN, D.R. Spatial variability of soil physical properties in the field. In: HILLEL, D., ed. Applications of soil physics. New York, Academic Press, 1980. p.319-344. 